OPEN ACCESS

Edited by:

Diana Imhof,

University of Bonn, Germany

Reviewed by:

Octavio Luiz Franco,

Catholic University of Brasilia, Brazil

Yuji Nishiuchi,

GlyTech, Inc., Japan

*Correspondence:

Zoltán Gáspár

gaspari.zoltan@itk.ppke.hu

Sándor Pongor

pongor@itk.ppke.hu

Specialty section:

This article was submitted to

Chemical Biology,

a section of the journal

Frontiers in Chemistry

Received: 19 December 2019 Accepted: 26 February 2020

Published: 17 March 2020

Citation:

Juhász J, Gáspári Z and Pongor S

(2020) Structure and Oxidative Folding

of AAl, the Major Alfa-Amylase

Inhibitor From Amaranth Seeds.

Front. Chem. 8:180

doi: 10.3389/fchem.2020.00180

\section{Structure and Oxidative Folding of AAl, the Major Alfa-Amylase Inhibitor From Amaranth Seeds}

\author{
János Juhász ${ }^{1,2}$, Zoltán Gáspári ${ }^{1 *}$ and Sándor Pongor ${ }^{1 *}$ \\ ${ }^{1}$ Faculty of Information Technology and Bionics, Pázmány Péter Catholic University, Budapest, Hungary, ${ }^{2}$ 3in-PPCU \\ Research Group, Faculty of Information Technology and Bionics, Pázmány Péter Catholic University, Esztergom, Hungary
}

AAl, the major alpha-amylase inhibitor (AAl) present in the seeds of the Mexican crop plant Amaranthus hypocondriacus is a 32-residue-long polypeptide with three disulfide bridges. Its structure is most closely related to the plant amylase inhibitor subfamily of knottins characterized by a topological knot formed by one disulfide bridge threading through a loop formed by the peptide chain as well as a short three-stranded beta sandwich core. AAl is specific against insect amylases and does not act on corresponding human or mammalian enzymes. It was found that the oxidative folding of AAl seems to follow a hirudine-like pathway with many non-native intermediates, but notably it proceeds through a major folding intermediate (MFI) that contains a vicinal disulfide bridge. Based on a review of the pertinent literature, the known vicinal disulfides in native proteins as well as well as the network of disulfide interchanges, we propose that MFI is a kinetic trap corresponding to a compact molten globule-like state which constrains the peptide chain to a smaller number of conformations that in turn can be rapidly funneled toward the native state.

Keywords: AAI, Amaranth alpha-amylase inhibitor, Amaranthus hypocondriacus, oxidative folding, folding intermediate, vicinal disulfide

\section{AAI: THE MAJOR ALPHA AMYLASE INHIBITOR OF Amaranthus hypochondriacus}

AAI (amaranth amylase inhibitor) is an alpha-amylase inhibitor isolated from the Mexican crop plant Amaranthus hypochondriacus. Amaranth grains are known to be cultivated for about 8,000 years and were part of the diet of the Aztecs. Today, Amaranth is grown in Mexico, Peru, and Bolivia, but the world's largest producer is China with an estimated annual production of 87 million metric tons. Amaranths are classified as pseudo cereals as they are not in the same botanical family as true cereals to which their grains bear similarity.

Aqueous extracts of Amaranth grains were found to inhibit insect alpha-amylases extracted from the larvae of the red flour beetle (Dibolium castaneum) and of the grain borer (Prostephanus truncatus). The alpha-amylase was purified with classical techniques (ChagollaLopez et al., 1994). Briefly, crude extracts of amaranth flour were fractionated by ammonium sulfate precipitation (35-65\%), fractionated on G75 Sepharose columns and the lyophilized active fractions were subjected to ion exchange chromatography The majority of inhibitory activity was found in one major peak which was further purified with reverse phase HPLC (RP-HPLC). The inhibitory activity was resistant to heat. As amino acid analysis revealed a high percentage of cysteine with no free sulfhydryl groups, the samples were reduced 
and pyridylethylated prior to sequencing. Digestion with trypsin and cyanogen bromide resulted in 7 overlapping peptides sequenced by automated Edman degradation which gave an assembled sequence of 32 amino acids with 6 cysteines and four prolines. The disulfide bridges were determined from partial double digests of the non-reduced peptide obtained with trypsin/chymotripsin. The disulfide topology could be determined except for the uncertainty caused by the vicinal cysteines 18 and 19, but a consensus topology could be predicted based on the multiple alignment (Figure 1) which was subsequently confirmed with both NMR (Lu et al., 1999) and X-ray crystallography (Pereira et al., 1999).

As shown by the NMR structure in Figure 2, AAI contains 3 disulfide bridges in the $a b c a b c$ topology, bridge $a$ connecting Cys 1 and Cys18, bridge $b$ connecting Cys 8 and Cys23, and bridge c connecting Cys17 and Cys31, respectively. With its length of only 32 residues, AAI was the shortest alpha-amylase inhibitor known at the time of its discovery, about 10 years later related amylase inhibitors of 30 amino acids were discovered (Tam et al., 2015). Purified AAI was found to be specific for insect amylases but not inhibiting mammalian amylases. This is an important property since many edible high protein seeds such as those of legumes contain enzyme inhibitors that are toxic to animals and humans and have to be destroyed by cooking or roasting.

\section{THE 3D STRUCTURE OF AAI}

The 3D structure of AAI was first predicted with molecular modeling based on homology to other peptides (Chagolla-Lopez et al., 1994). Namely, multiple alignment (Figure 1) revealed that AAI is homologous to knottin proteins noted for a pseudoknot formed by a conserved disulfide arrangement found in a family of short peptides. The term was coined-as far as we know-a few years before the isolation of AAI (Le Nguyen et al., 1990). As of today there is well-maintained database of knottin structures (knottin dbase) that currently has 3,320 sequences and 214 3D structure entries (Postic et al., 2018) (www. dsimb.inserm.fr/KNOTTIN/). Based on the abundant structural

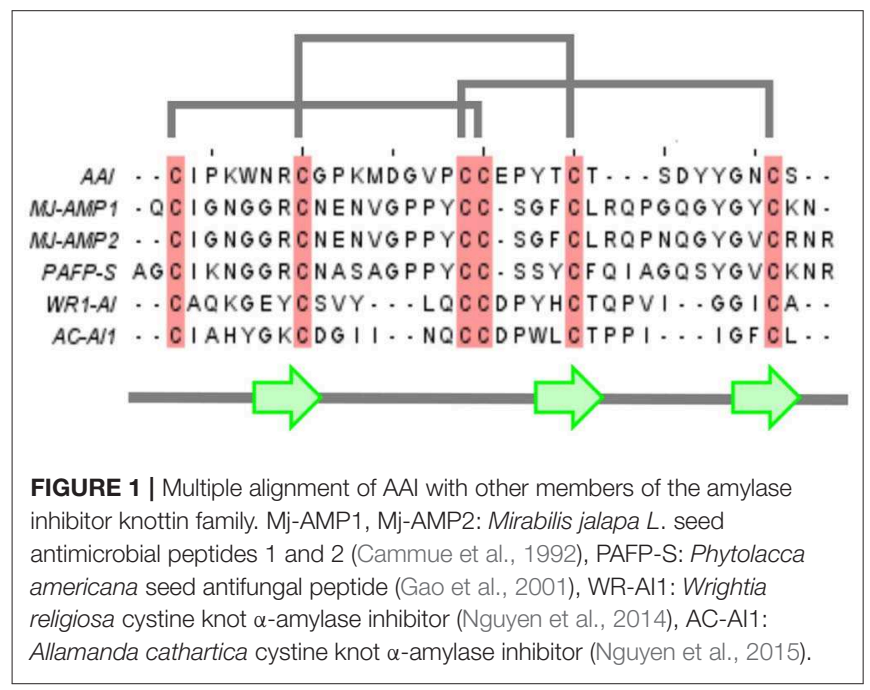

information available today, we can safely conclude that AAI belongs to a specific subclass of knottins, the plant alfa- amylase inhibitors (Tam et al., 2015) that contain a short beta sandwich of three (sometimes only two) beta strands, the third strand being sometimes less regular due to the shortness of the sandwich. The short beta sandwich was included into the first predicted structure of AAI which in this way turned out to be analogous to the consensus structure of knottins, more exactly to the subgroup of plant alpha-amylase inhibitor knottins defined later (Tam et al., 2015).

The X-ray structure of AAI, in complex with the $\alpha$-amylase of yellow meal worm (Tenebrio molitor) larvae (TMA) was determined at $2.0 \AA$ resolution (Pereira et al., 1999). In addition to confirming the knottin-like structure, it was found that AAI binds to the active-groove of TMA via two segments, the first and the last intercysteine loops. According to the X-ray structure, TMA-bound AAI blocks the central four sugar-binding subsites of TMA rendering substrate binding impossible (Figure 3). In addition, molecular docking techniques were used predict the

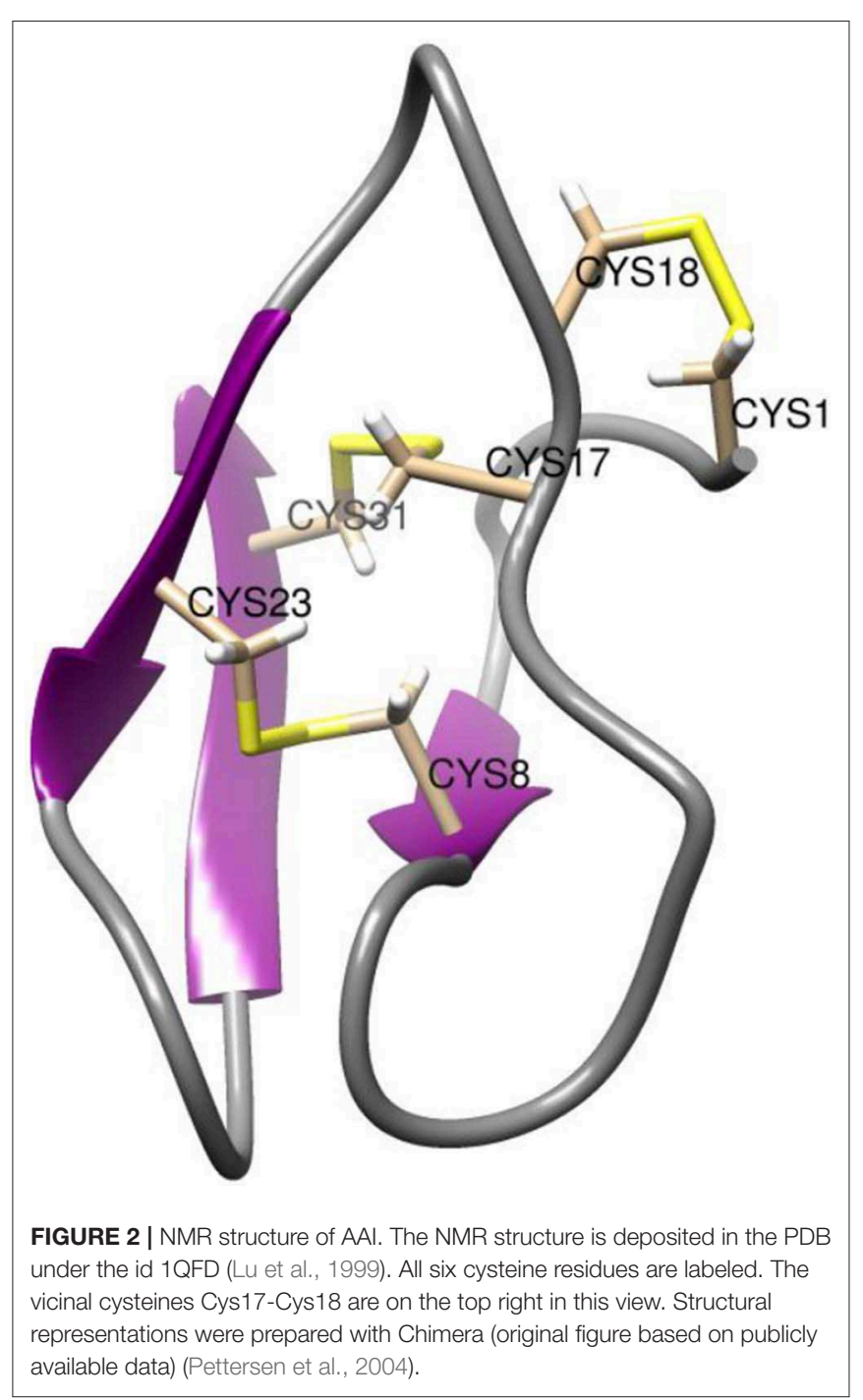




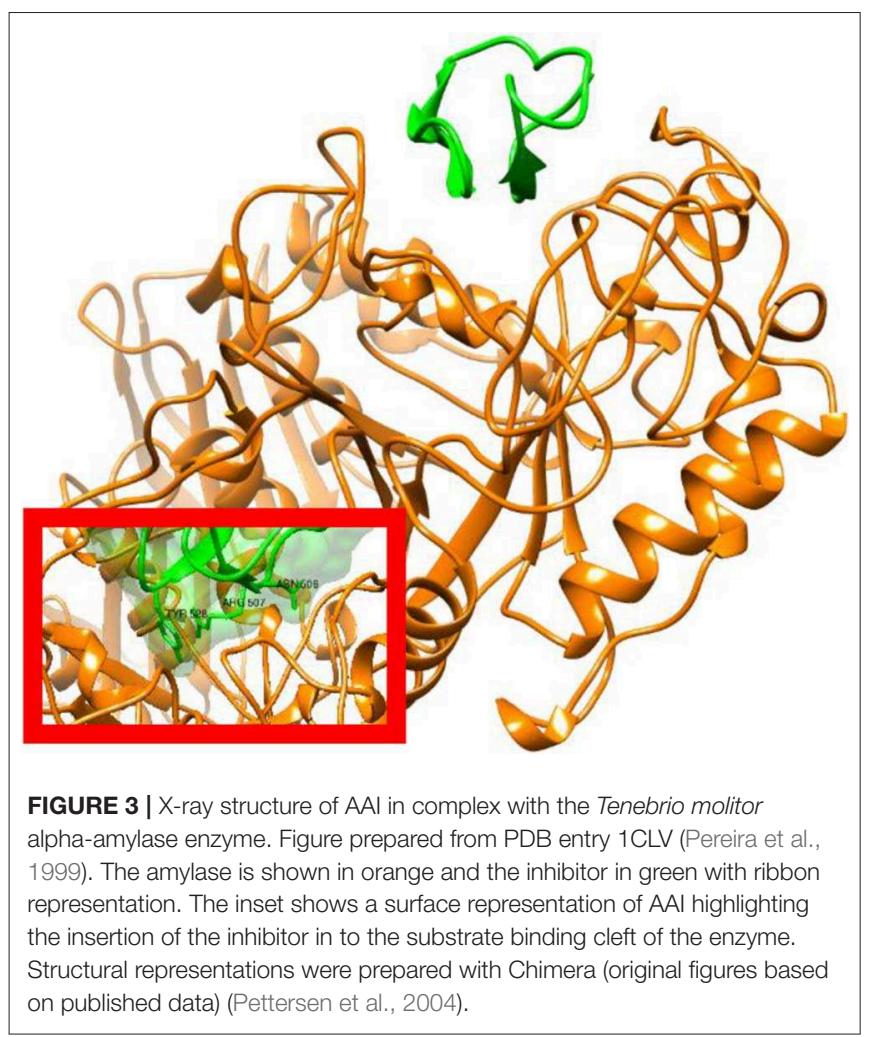

binding of AAI to porcine pancreatic alpha-amylase (PPA). It was found AAI can form only 8 direct (not solvent-mediated) hydrogen bonds to the porcine enzyme as opposed to the 14 such TMA, which provides a simple molecular explanation to the inhibitory specificity of AAI toward insect amylases.

\section{SYNTHESIS AND IN VITRO OXIDATIVE FOLDING OF AAI}

The AAI peptide was synthesized manually on a 1-mmol scale by solid-phase peptide synthesis using an Fmoc (N(9-fluorenyl)methoxycarbonyl) methodology (Lozanov et al., 1997; Cemazar et al., 2003). The crude peptide was isolated with yield $90 \%$ and purified to homogeneity ( $98 \%$, RP-HPLC). Disulfide bridges were formed by oxidative folding in a cysteine $(1 \mathrm{mM})$-cystine $(0.05 \mathrm{mM})$ redox buffer containing $1 \mathrm{M}$ guanidine hydrochloride. The reaction was left to proceed at room temperature for $16^{\mathrm{h}}$, the overall yield of the HPLC purified peptide was $>90 \%$. The synthetic product had the same physicochemical and enzyme inhibitory properties as the natural product. In addition, several orthogonal cysteine protection schemes were tried, in which the disulfide bridges were produced in a well-defined order but active inhibitors were not obtained. It was concluded that "... oxidative folding of AAI may not be a simple process but may rather proceed via transient intermediates, i.e., through closing and opening of disulfide bonds" (Lozanov et al., 1997).

The oxidative folding of AAI was studied with a variety of techniques [acid quenching followed by RP-HPLC and mass

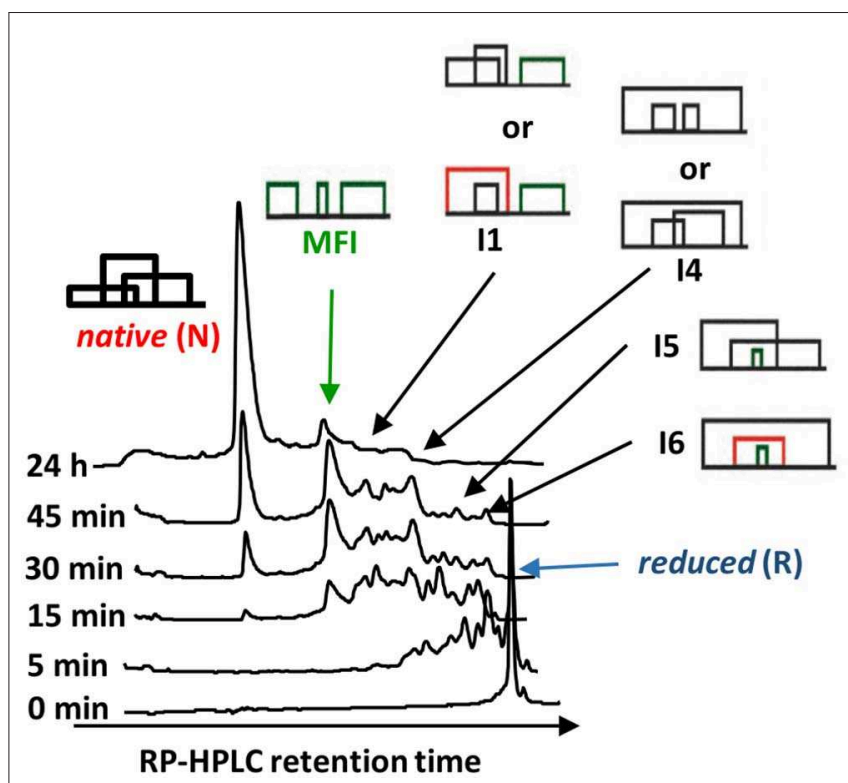

FIGURE 4 | The oxidative folding intermediates of AAI. Oxidative folding was carried out at pH $8.5,25^{\circ} \mathrm{C}$, by placing $100 \mathrm{mg}_{-}$liter ${ }^{-1} \mathrm{AAl}$ into the refolding buffer (100 mM ammonium acetate/1 M guanidinium hydrochloride/1 mM cysteine/ $0.05 \mathrm{mM}$ cystine/2 $\mathrm{mM}$ EDTA). The same distribution of intermediates was observed when the folding was carried out at different $\mathrm{pH}$ values $(7.0,7.5$, 8.0, and 8.5) and in the absence of guanidinium hydrochloride. The disulfide connectivity of the intermediates was determined by acid trapping and mass spectrometry as described in the text. The disulfide pairings of intermediates 11 and 14 could not be unequivocally determined due to the vicinal cysteines.

spectrometry, NMR, photoCIDNP [photochemically induced dynamic nuclear polarization], CD [circular dichroism]] (Cemazar et al., 2003, 2004). The conditions included the synthesis conditions mentioned above as well as an enzyme catalyzed folding carried out in the presence of bacterial disulfide isomerase DsBC In most of the cases, a fixed group of 5 fully oxidized intermediates were identified (Figure 4). All but two of the observed disulfide bridges were non-native, and a non-native vicinal disulfide bond between cysteines 17 and 18 was present in three of the intermediates (Figure 4). Because of the vicinal cysteines, the disulfide connectivity could not be unequivocally determined in the case of I1 and I4, so we assigned alternative structures to both of them (I1-1, I1-2 and I4-1, I4-2, respectively). The time course of oxidative folding shows a further peculiarity, i.e., a dominant, major folding intermediate (MFI) that also contained the vicinal disulfide bridge was transiently present (Figure 5). The position of MFI within the chromatogram shows that MFI is relatively hydrophilic as compared to the reduced species which suggests that the hydrophobic residues may be more buried than in the reduced species. Even though native AAI and MFI possess different disulfide pairings, their sizes are indistinguishable within the experimental error (Cemazar et al., 2004). This makes us believe that MFI may play a role similar to that of the molten globule state of larger proteins by constraining the peptide chain to a smaller number of conformations that can be rapidly funneled toward the native state. 


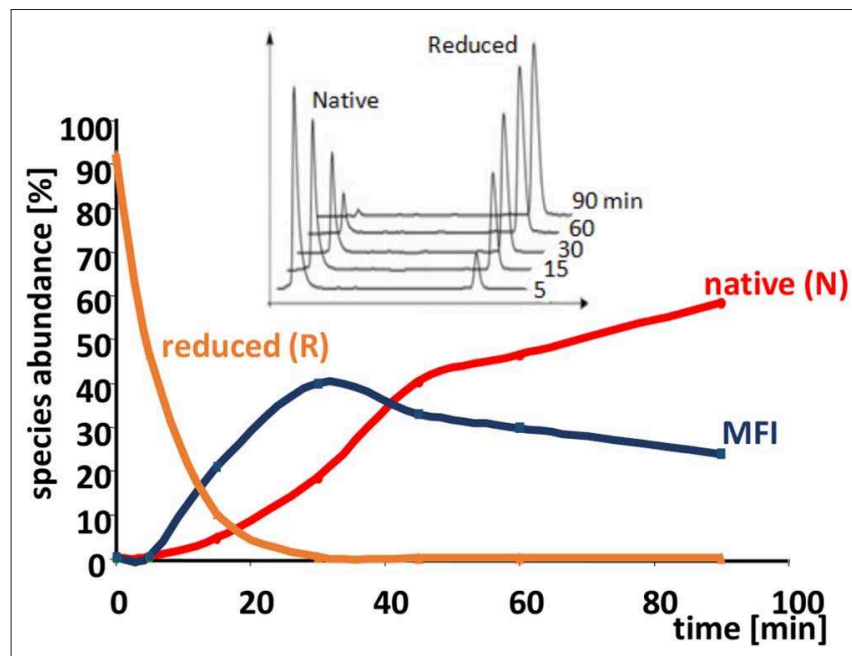

FIGURE 5 | Time course of the oxidative folding of AAl. The abundance of reduced and native forms, as well as the MFI are shown as a function of time during folding. The accumulation of the MFI and then its gradual conversion to the native form is clearly visible. Inset: reductive unfolding of AAl carried out with dithio-treitol (DTT) shows an all-or-none mechanism i.e., no intermediates are shown in addition to the native and the completely reduced species. The reduction was carried out in a buffer similar to that described in the legend to Figure 4 except that it contained $1-10 \mathrm{mM}$ of DTT instead of cysteine/cystine (original figure based on published data).

The dominance of MFI was observed both for the enzyme catalyzed and for the cysteine/cystine catalyzed reaction, the only difference was that in the enzyme catalyzed reaction intermediate I1 was the most intensive for the first minutes of the folding process (data not shown). The disulfide connectivity of MFI is "bead-like" i.e., Cys residues pair with their sequential neighbors. This is in good accordance with the general view that local interactions dominate the first stages of protein folding. In order to get insights into the role the intermediates we prepared a folding map with intermediates as the nodes and disulfide exchange reactions as the edges (Agoston et al., 2005). The logics underlying this representation is that fully oxidized intermediates such as observed for AAI can undergo intramolecular disulfide interchange reactions that rewire the two participating disulfide bridges (example shown in Figure 6). The full network of the intermediates has 15 nodes representing fully oxidized intermediates and 45 edges each representing a rearrangement analogous to that showed in Figure 6. If we map the observed intermediates on this network, we get a folding map shown in Figure 7. One can notice that (i) the network of observed intermediates is small i.e., there is a short route between the intermediates, (ii) MFI plays a central role in this small network as it is connected to 4 out of 6 observed intermediates; and finally, (iii) the native state is accessible via two intermediates, and is only two reshuffling steps away from MFI.

NMR and photoCIDNP experiments revealed that the reduced form $\mathrm{R}$ is close to random coil, but MFI has some structure (or rather a set of slowly interconverting structures) that differs from that of the native AAI especially in terms

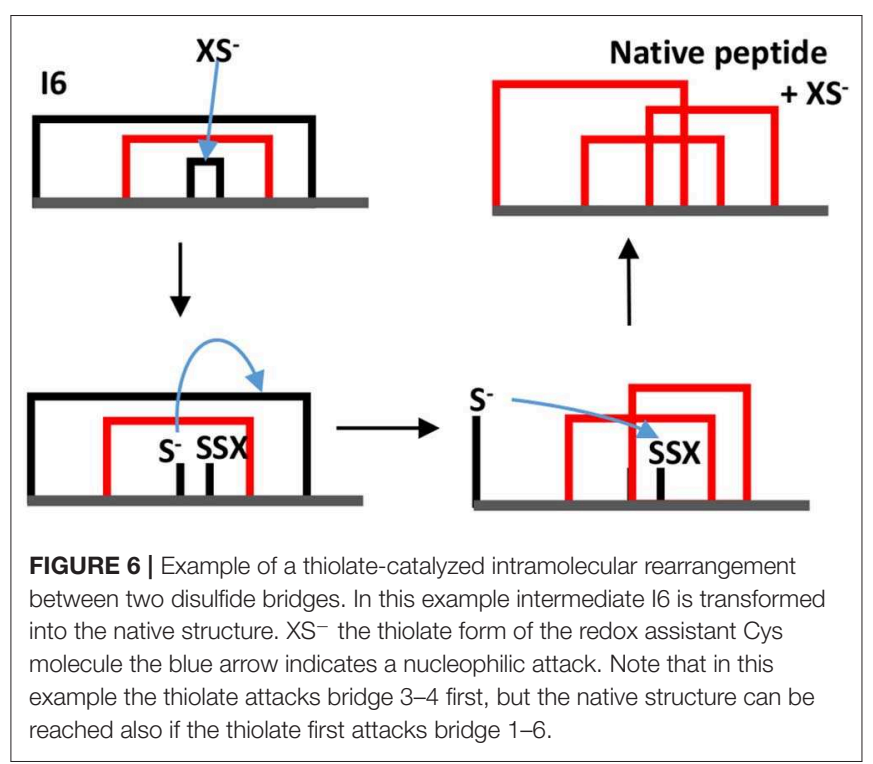

of the accessibility of aromatic side chains that is revealed by photoCIDNP. Time resolved NMR revealed a monotonous change in the aliphatic and aromatic NMR signals, respectively, and confirmed that enzyme catalyzed reaction was somewhat faster, even though it proceeded through the same intermediates, with minor quantitative differences at the first stages of the reaction (Carugo et al., 2003).

\section{THE ROLE OF THE VICINAL DISULFIDE BRIDGE}

Chemical intuition suggests that a vicinal S-S bond (and the associated eight-member ring) could have structural effects similar to a proline residue (a five-member ring) in constraining the movement of the main chain in such a way that a turn is formed (Carugo et al., 2003). Or, as Jane Richardson and associates put it: "A vicinal disulfide acts conformationally rather like a super-sized proline ring that can rigidly organize a connected region of side chains and backbone" (Richardson et al., 2017). About the molecular details of the 8 membered rings there is a variety of opinions. Earlier studies pointed out that the peptide bond of the vicinal disulfide bridge is trans and it imposes a turn like structure (Carugo et al., 2003). A detailed theoretical study suggested that cis-amide containing rings are also stable, although no examples were found in the databases at the time (Hudáky et al., 2004). A more complete statistical overview of current databases suggests that vicinal disulfide turns form 3 distinct clusters, two corresponding to trans amide bond, one to cis (Figure 8). The cis vicinal structures depicted on Figure 9 are in fact closest to type VIa turns, but, in agreement with the theoretical calculations (Hudáky et al., 2004), the C $\alpha$ distance criterion of $<7 \AA$ is not met. On the other hand, the majority of the vicinal disulfides belong to the other clusters that resemble a type VIII turn with a distorted trans peptide bond between the two cysteines, with omega torsion angles ranging from $-176^{\circ}$ to 


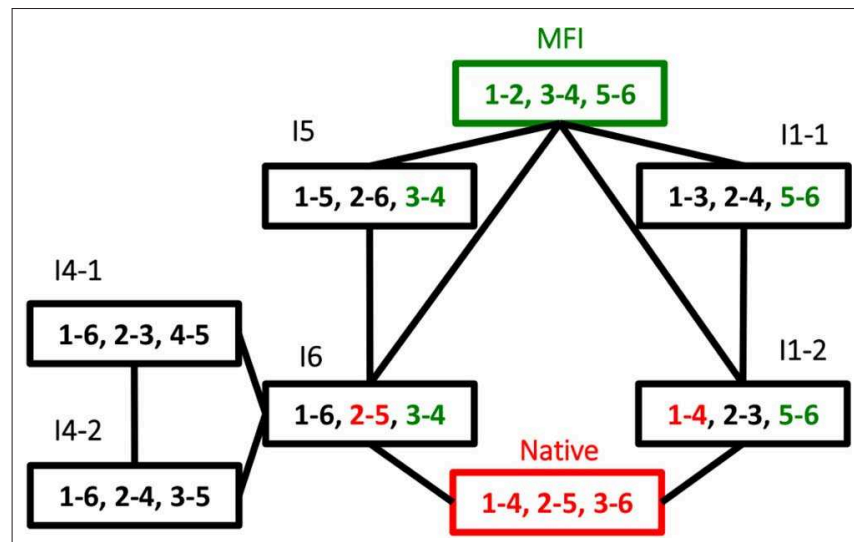

FIGURE 7 | Oxidative folding map built from the disulfide intermediates observed oxidative folding of AAl. Each node is an intermediate, folding map of the observed disulfide intermediates shown in Figure 4. The nodes are the observed intermediates, numbers indicate the serial order of cysteine residues within the sequence, i.e., $1=$ Cys 1,2 = Cys8, 3 = Cys 17, $6=$ Cys30. The edges are intramolecular folding reactions (reshuffling reactions), an example for them is shown in Figure 6.

$-152^{\circ}$ with an average of around $-162^{\circ}$ in our demonstrative data set. Taken together, the pronounced difference observed between the oxidized and reduced states, suggests that vicinal disulfides may act "as a 'redox-activated' conformational switch" (Carugo et al., 2003).

\section{SUMMARY AND CONCLUSIONS}

Oxidative folding can be best pictured as the fusion of two competing processes, the formation of covalent disulfide bridges on the one hand, and the formation the non-covalent interactions on the other hand, that are known to give rise to secondary and tertiary structure. It is believed that there are two extreme pathway types of oxidative folding (Narayan et al., 2000; Chang, 2011). The one nicknamed BPTI-like after bovine pancreatic trypsin inhibitor, is dominated by conformational folding, and as a consequence, only (or mostly) native disulfide bridges are found among the observable intermediates. In contrast, "hirudin-type" folding is dominated by disulfide formation and is characterized by a variety of intermediates with mostly nonnative disulfide bridges. AAI clearly falls into this latter group, based on the predominance of non-native disulfide bonds in its folding intermediates as well as on the all-or-none type reductive unfolding profile characteristic of this group (Figure 5, inset). As the intermediates seem to be closely related (Figure 7) we tend to believe that AAI can form via a variety of similar pathways, i.e., there may be no single folding pathway that could be clearly distinguished as compared to the others. The dominance of MFI in the process can be explained by analogies to the familiar concepts of protein folding: (i) The beadlike disulfide topology corresponds to local connections that form in the early phases of the conformational folding process. To this we add, that an similar bead-like structure is the most abundant intermediate during the oxidative folding of hirudin

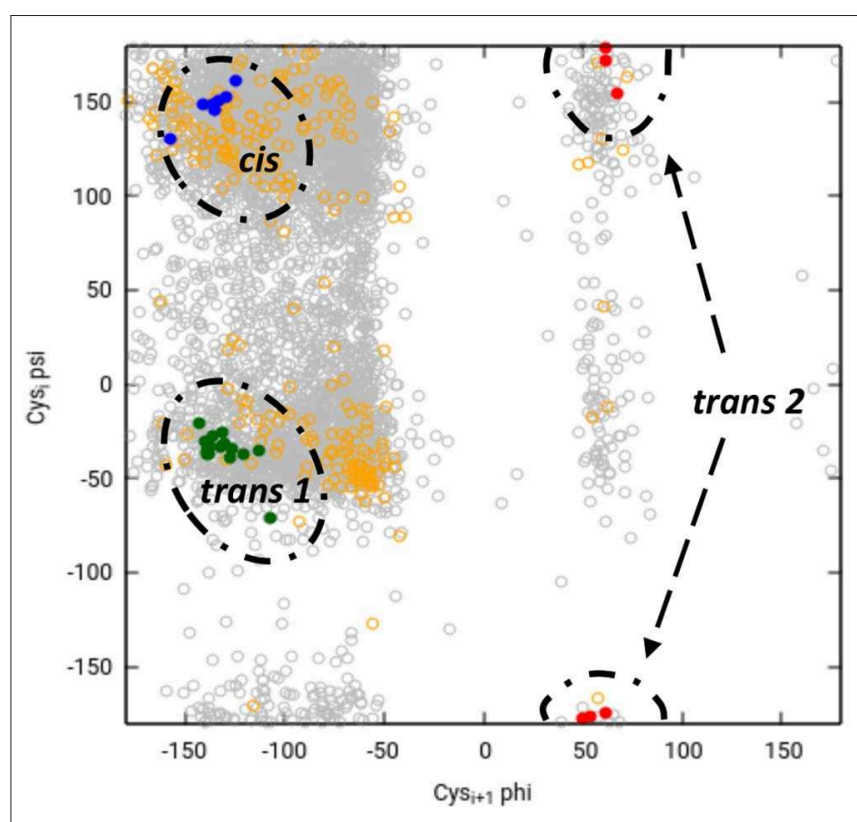

FIGURE 8 | Ramachandran-like representation of vicinal disulfide bridges in current 3DB databases. Data from the Cys.sqlite database (Fobe et al., 2019) filtered according to the CATH 40\% non-redundant list (Dawson et al., 2017). Gray circles represent all disulfide-forming cysteines, orange circles depict all neighboring cysteines that are not linked to each other. Vicinal disulfide bridges with cis amide bonds between the cysteines are shown with blue dots, whereas the two clusters with trans amide bonds are colored green (cluster 1) and red (cluster 2). Note that due to the " $-180^{\circ}-180^{\circ}$ " convention of the Ramachandran map, cluster 2 is split and appears at the top and the bottom.

core domain (Chang, 2011), (ii) The compact, hydrophilic form (apparent from NMR and RP-HPLC, respectively) as well as the slowly interchanging conformations (NMR) are reminiscent of the molten globule-like state observed in protein folding, (iii) The centrally located vicinal disulfide turn may help the molecule to get into a compact form. Intermediate I6 contains two further disulfide bonds that fix the chain in a roughly $\mathrm{U}$-shaped form. Interestingly, this disulfide arrangement is analogous to the ladder-like intermediate seen in the folding of various other knottins (for reviews see Cemazar et al., 2008; Reinwarth et al., 2013). Summarizing it appears that MFI forms via aspecific collapse and is consolidated into the native state via intramolecular disulfide reshuffling reactions.

To our knowledge, the biotechnological potential of AAI-like proteins is yet a largely unexplored area. Although there are a number of disupfide-rich peptides that are extensively used in protein design as scaffolds (Wang and Craik, 2018) AAI is yet to be explored in this respect. Although defensin-like peptides, containing vicinal cysteines, are among the successful examples where designed disulfide pairings can be synthetically achieved (Zheng et al., 2017) the possible utilization of a folding route with a transient vicinal disulfide has not been investigated. We propose that future protein design attempts could make use of the large interaction surface of AAI as well as its unique folding mechanism to explore novel possible applications and synthetic routes. 

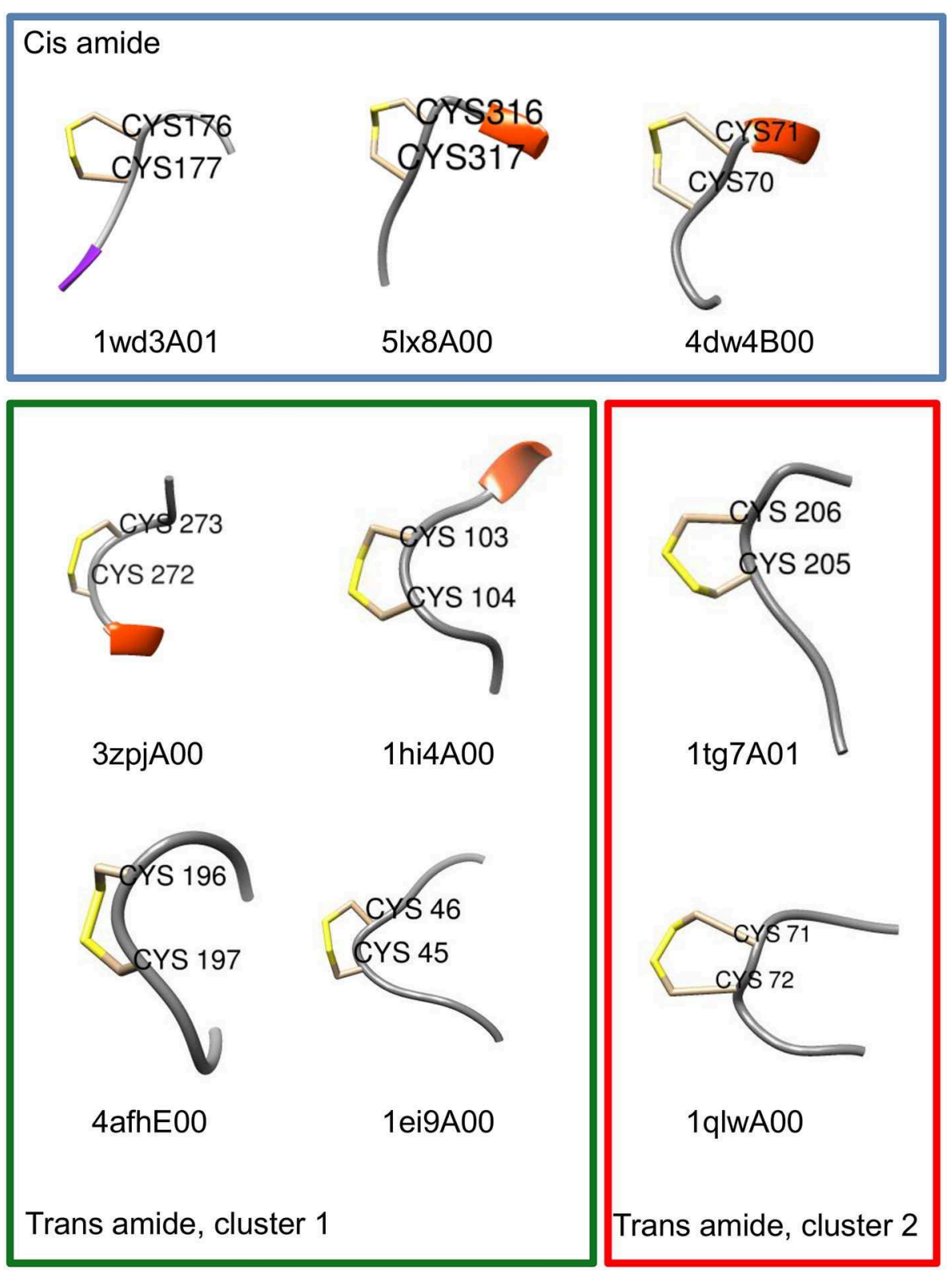

FIGURE 9 | Examples of turn-like conformations induced by vicinal disulfide bridges. Examples for the cis and trans clusters are shown with CATH domain codes. Six residue fragments re shown with positions 3 and 4 corresponding to the disulfide-forming vicinal cysteines. Structural representations were prepared with Chimera (Pettersen et al., 2004). Frame coloring corresponds to the cluster colors in Figure 8.

\section{AUTHOR CONTRIBUTIONS}

JJ, ZG, and SP designed the review and drafted the manuscript.

\section{FUNDING}

This project was supported in part by the Hungarian government grants OTKA 120650 (Microbiome bioinformatics:
Computational analysis of complex bacterial communities) EFOP-3.6.2-16-2017-00013 (4. Integration of high-throughput biological data), EFOP-3.6.3-VEKOP-16-2017-00002 (2. Nonconventional computing and modeling approaches) which were supported by the European Union and co-financed by the European Social Fund as well as by grant ED_17-1-2017-0009 of the National Bionics Program sponsored by Hungarian Ministry of Technology and Innovation. 


\section{ACKNOWLEDGMENTS}

The authors thank for the help and advice of Drs. András Patthy, Sotir Zahariev, Corrado Gurnaccia,

\section{REFERENCES}

Agoston, V., Cemazar, M., Kaján, L., and Pongor, S. (2005). Graphrepresentation of oxidative folding pathways. BMC Bioinform. 6:19. doi: 10.1186/1471-2105-6-19

Cammue, B. P., De Bolle, M. F., Terras, F. R., Proost, P., Van Damme, J., Rees, S. B., et al. (1992). Isolation and characterization of a novel class of plant antimicrobial peptides form mirabilis Jalapa L. Seeds. J. Biol. Chem. 267, 2228-2233.

Carugo, O., Cemazar, M., Zahariev, S., Hudáky, I., Gáspári, Z., Perczel, A., et al. (2003). Vicinal disulfide turns. Protein. Eng. 16, 637-639. doi: $10.1093 /$ protein/gzg088

Cemazar, M., Gruber, C. W., Craik, D. J. (2008). Oxidative folding of cyclic cystine knot proteins. Antioxid. Redox Signal. 10, 103-111. doi: 10.1089/ars.2007.1849

Cemazar, M., Zahariev, S., Lopez, J. J., Carugo, O., Jones, J. A., Hore, P. J., et al. (2003). Oxidative folding intermediates with nonnative disulfide bridges between adjacent cysteine residues. Proc. Natl. Acad. Sci. U.S.A. 100, 5754-5759. doi: 10.1073/pnas.2225470100

Cemazar, M., Zahariev, S., Pongor, S., and Hore, P. J. (2004). Oxidative folding of amaranthus $\alpha$-amylase inhibitor: disulfide bond formation and conformational folding. J. Biol. Chem. 279, 16697-16705. doi: 10.1074/jbc.M312328200

Chagolla-Lopez, A., Blanco-Labra, A., Patthy, A., Sánchez, R., and Pongor, S. (1994). A novel $\alpha$-amylase inhibitor from amaranth (Amaranthus hypocondriacus) seeds. J. Biol. Chem. 269, 23675-23680.

Chang, J-Y. (2011). Diverse pathways of oxidative folding of disulfide proteins, underlying causes and folding models. Biochemistry 50, 3414-3431. doi: $10.1021 /$ bi200131j

Dawson, N. L., Lewis, T. E., Das, S., Lees, J. G., Lee, D., Ashford, P., et al. (2017). CATH: an expanded resource to predict protein function through structure and sequence. Nucleic Acids Res. 45, D289-D295. doi: 10.1093/nar/gkw1098

Fobe, T. L., Kazakov, A., and Riccardi, D. (2019). Cys.sqlite: a structuredinformation approach to the comprehensive analysis of cysteine disulfide bonds in the protein databank. J. Chem. Inf. Model. 59, 931-943. doi: $10.1021 /$ acs.jcim. 8 b00950

Gao, G. H., Liu, W., Dai, J. X., Wang, J. F., Hu, Z., Zhang, Y., et al. (2001). Solution structure of PAFP-S: a New Knottin-type antifungal peptide from the seeds of phytolacca americana. Biochemistry 40, 10973-10978. doi: 10.1021/bi010167k

Hudáky, I., Gáspári, Z., Carugo, O., Cemažar, M., Pongor, S., and Perczel, A. (2004). Vicinal disulfide bridge conformers by experimental methods and by ab initio and DFT molecular computations. Proteins Struct. Func. Gene 55, 152-168. doi: 10.1002/prot.10581

Le Nguyen, D., Heitz, A., Chiche, L., Castro, B., Boigegrain, R. A., Favel, A., et al. (1990). Molecular recognition between serine proteases and new bioactive microproteins with a knotted structure. Biochimie 72, 431-435. doi: 10.1016/0300-9084(90)90067-Q

Lozanov, V., Guarnaccia, C., Patthy, A., Foti, S., and Pongor, S. (1997). Synthesis and cystine/cysteine-catalyzed oxidative folding of the amaranth $\alpha$-amylase inhibitor. J. Pept. Res. 50, 65-72. doi: 10.1111/j.1399-3011.1997.tb00621.x

Lu, S., Deng, P., Liu, X., Luo, J., Han, R., Gu, X., et al. (1999). Solution structure of the major $\alpha$-amylase inhibitor of the crop plant amaranth. J. Biol. Chem. 274, 20473-20478. doi: 10.1074/jbc.274.29.20473
Maša Čemažar, Stefan Strobl, Profs. Peter J. Hore, Robert Huber, and the late Prof. X. Gu. The comments of Dr. E. Welker on the manuscript are gratefully acknowledged.
Narayan, M., Welker, E., Wedemeyer, W. J., and Scheraga, H. A. (2000). Oxidative folding of proteins. Acc. Chem. Res. 33, 805-812. doi: 10.1021/ar000063m

Nguyen, P. Q, Luu, T. T., Bai, Y., Nguyen, G. K., Pervushin, K., and Tam, J. P. (2015). Allotides: proline-rich cystine knot $\alpha$-amylase inhibitors from allamanda cathartica. J. Nat. Prod. 78, 695-704. doi: 10.1021/np500866c

Nguyen, P. Q., Wang, S., Kumar, A., Yap, L. J., Luu, T. T., and Lescar, J. (2014). Discovery and characterization of pseudocyclic cystine-knot $\alpha$-amylase inhibitors with high resistance to heat and proteolytic degradation. FEBS J. 281, 4351-4366. doi: 10.1111/febs.12939

Pereira, P. J., Lozanov, V., Patthy, A., Huber, R., Bode, W., Pongor, S. et al. (1999). Specific inhibition of insect $\alpha$-amylases: yellow meal worm $\alpha$-amylase in complex with the amaranth $\alpha$-amylase inhibitor at 2.0 å resolution. Structure 7, 1079-1088. doi: 10.1016/S0969-2126(99)80175-0

Pettersen, E. F., Goddard, T. D., Huang, C. C., Couch, G. S., Greenblatt, D. M., Meng, E. C. et al. (2004). UCSF chimera - a visualization system for exploratory research and analysis. J. Comput. Chem. 25, 1605-1612. doi: 10.1002/jcc.20084

Postic, G., Gracy, J., Périn, C., Chiche, L., and Gelly, J. C. (2018). KNOTTIN: the database of inhibitor cystine knot scaffold after 10 years, toward a systematic structure modeling. Nucleic Acids Res. 46, D454-D458. doi: $10.1093 / \mathrm{nar} / \mathrm{gkx} 1084$

Reinwarth, M., Glotzbach, B., Tomaszowski, M., Fabritz, S., Avrutina, O., and Kolmar, H. (2013). Oxidative folding of peptides with cystine-knot architectures: kinetic studies and optimization of folding conditions. ChemBioChem 14, 137-146. doi: 10.1002/cbic.2012 00604

Richardson, J. S, Videau, L. L., Williams, C. J., and Richardson, D. C. (2017). Broad analysis of vicinal disulfides: occurrences, conformations with cis or with trans peptides, and functional roles including sugar binding. J. Mol. Biol. 429, 1321-1335. doi: 10.1016/j.jmb.2017.03.017

Tam, J. P, Wang, S., Wong, K. H., and Tan, W. L. (2015). Antimicrobial peptides from plants. Pharmaceuticals 8, 711-757. doi: 10.3390/ph80 40711

Wang, C. K., and Craik, D. J. (2018). Designing macrocyclic disulfide-rich peptides for biotechnological applications. Nat. Chem. Biol. 14, 417-427. doi: 10.1038/s41589-018-0039-y

Zheng, Y., Li, Z., Ren, J., Liu, W., Wu, Y., Zhao, Y., et al. (2017). Artificial disulfide-rich peptide scaffolds with precisely defined disulfide patterns and a minimized number of isomers. Chem. Sci. 8, 2547-2552. doi: 10.1039/C6SC0 $5710 \mathrm{~A}$

Conflict of Interest: The authors declare that the research was conducted in the absence of any commercial or financial relationships that could be construed as a potential conflict of interest.

Copyright (๑) 2020 Juhász, Gáspári and Pongor. This is an open-access article distributed under the terms of the Creative Commons Attribution License (CC BY). The use, distribution or reproduction in other forums is permitted, provided the original author(s) and the copyright owner(s) are credited and that the original publication in this journal is cited, in accordance with accepted academic practice. No use, distribution or reproduction is permitted which does not comply with these terms. 